\title{
Ceramide turnover (version 2019.4) in the IUPHAR/BPS Guide to Pharmacology Database
}

\author{
Anthony $\mathrm{H}$. Futerman ${ }^{1}$
}

1. Weizmann Institute of Science, Israel

\begin{abstract}
Ceramides are a family of sphingophospholipids synthesized in the endoplasmic reticulum, which mediate cell stress responses, including apoptosis, autophagy and senescence, Serine palmitoyltransferase generates 3ketosphinganine, which is reduced to sphinganine (dihydrosphingosine). N-Acylation allows the formation of dihydroceramides, which are subsequently reduced to form ceramides. Once synthesized, ceramides are trafficked from the ER to the Golgi bound to the ceramide transfer protein, CERT (COL4A3BP, Q9Y5P4). Ceramide can be metabolized via multiple routes, ensuring tight regulation of its cellular levels. Addition of phosphocholine generates sphingomyelin while carbohydrate is added to form glucosyl- or galactosylceramides. Ceramidase re-forms sphingosine or sphinganine from ceramide or dihydroceramide. Phosphorylation of ceramide generates ceramide phosphate. The determination of accurate kinetic parameters for many of the enzymes in the sphingolipid metabolic pathway is complicated by the lipophilic nature of the substrates.
\end{abstract}

\section{Contents}

This is a citation summary for Ceramide turnover in the Guide to Pharmacology database (GtoPdb). It exists purely as an adjunct to the database to facilitate the recognition of citations to and from the database by citation analyzers. Readers will almost certainly want to visit the relevant sections of the database which are given here under database links.

GtoPdb is an expert-driven guide to pharmacological targets and the substances that act on them. GtoPdb is a reference work which is most usefully represented as an on-line database. As in any publication this work should be appropriately cited, and the papers it cites should also be recognized. This document provides a citation for the relevant parts of the database, and also provides a reference list for the research cited by those parts.

Please note that the database version for the citations given in GtoPdb are to the most recent preceding version in which the family or its subfamilies and targets were substantially changed. The links below are to the current version. If you need to consult the cited version, rather than the most recent version, please contact the GtoPdb curators.

\section{Database links}

Ceramide turnover

http://www.guidetopharmacology.org/GRAC/FamilyDisplayForward?familyld=767

Serine palmitoyltransferase 
http://www.guidetopharmacology.org/GRAC/FamilyDisplayForward?familyld=788

Enzymes

SPT1(serine palmitoyltransferase long chain base subunit 1)

http://www.guidetopharmacology.org/GRAC/ObjectDisplayForward?objectld=2509

SPT2(serine palmitoyltransferase long chain base subunit 2)

http://www.guidetopharmacology.org/GRAC/ObjectDisplayForward?objectld=2510

SPT3(serine palmitoyltransferase long chain base subunit 3)

http://www.guidetopharmacology.org/GRAC/ObjectDisplayForward?objectld=2511

SPTSSA(serine palmitoyltransferase small subunit A)

http://www.guidetopharmacology.org/GRAC/ObjectDisplayForward?objectld=2512

SPTSSB(serine palmitoyltransferase small subunit B)

http://www.guidetopharmacology.org/GRAC/ObjectDisplayForward?objectld=2513

3-ketodihydrosphingosine reductase

http://www.guidetopharmacology.org/GRAC/FamilyDisplayForward?familyld=791

Enzymes

3-ketodihydrosphingosine reductase

http://www.guidetopharmacology.org/GRAC/ObjectDisplayForward?objectld=2463

Ceramide synthase

http://www.guidetopharmacology.org/GRAC/FamilyDisplayForward?familyld=789

Enzymes

CERS1 (ceramide synthase 1)

http://www.guidetopharmacology.org/GRAC/ObjectDisplayForward?objectld=2474

CERS2(ceramide synthase 2)

http://www.guidetopharmacology.org/GRAC/ObjectDisplayForward?objectld=2475

CERS3(ceramide synthase 3)

http://www.guidetopharmacology.org/GRAC/ObjectDisplayForward?objectld=2476

CERS4(ceramide synthase 4)

http://www.guidetopharmacology.org/GRAC/ObjectDisplayForward?objectld=2477

CERS5(ceramide synthase 5)

http://www.guidetopharmacology.org/GRAC/ObjectDisplayForward?objectld=2478

CERS6(ceramide synthase 6)

http://www.guidetopharmacology.org/GRAC/ObjectDisplayForward?objectld=2479

Sphingolipid $\Delta^{4}$-desaturase

http://www.guidetopharmacology.org/GRAC/FamilyDisplayForward?familyld=790

Enzymes

delta 4-desaturase, sphingolipid 1

http://www.guidetopharmacology.org/GRAC/ObjectDisplayForward?objectld=2484

delta 4-desaturase, sphingolipid 2

http://www.guidetopharmacology.org/GRAC/ObjectDisplayForward?objectld=2485

Sphingomyelin synthase

http://www.guidetopharmacology.org/GRAC/FamilyDisplayForward?familyld=774

\section{Enzymes}

sphingomyelin synthase 1

http://www.guidetopharmacology.org/GRAC/ObjectDisplayForward?objectld=2520

sphingomyelin synthase 2

http://www.guidetopharmacology.org/GRAC/ObjectDisplayForward?objectld=2521

sterile alpha motif domain containing 8

http://www.guidetopharmacology.org/GRAC/ObjectDisplayForward?objectld=2525

Sphingomyelin phosphodiesterase

http://www.guidetopharmacology.org/GRAC/FamilyDisplayForward?familyld=773 


\section{Enzymes}

sphingomyelin phosphodiesterase 1

http://www.guidetopharmacology.org/GRAC/ObjectDisplayForward?objectld=2514

sphingomyelin phosphodiesterase 2

http://www.guidetopharmacology.org/GRAC/ObjectDisplayForward?objectld=2515

sphingomyelin phosphodiesterase 3

http://www.guidetopharmacology.org/GRAC/ObjectDisplayForward?objectld=2516

sphingomyelin phosphodiesterase 4

http://www.guidetopharmacology.org/GRAC/ObjectDisplayForward?objectld=2517

sphingomyelin phosphodiesterase acid-like $3 \mathrm{~A}$

http://www.guidetopharmacology.org/GRAC/ObjectDisplayForward?objectld=2518

sphingomyelin phosphodiesterase acid-like 3B

http://www.guidetopharmacology.org/GRAC/ObjectDisplayForward?objectld=2519

Neutral sphingomyelinase coupling factors

http://www.guidetopharmacology.org/GRAC/FamilyDisplayForward?familyld=772

\section{Enzymes}

embryonic ectoderm development

http://www.guidetopharmacology.org/GRAC/ObjectDisplayForward?objectld=2487

neutral sphingomyelinase activation associated factor

http://www.guidetopharmacology.org/GRAC/ObjectDisplayForward?objectld=2495

Ceramide glucosyltransferase

http://www.guidetopharmacology.org/GRAC/FamilyDisplayForward?familyld=775

Enzymes

UDP-glucose ceramide glucosyltransferase

http://www.guidetopharmacology.org/GRAC/ObjectDisplayForward?objectld=2528

\section{Acid ceramidase}

http://www.guidetopharmacology.org/GRAC/FamilyDisplayForward?familyld=769

\section{Enzymes}

$\mathrm{N}$-acylsphingosine amidohydrolase 1

http://www.guidetopharmacology.org/GRAC/ObjectDisplayForward?objectld=2491

Neutral ceramidases

http://www.guidetopharmacology.org/GRAC/FamilyDisplayForward?familyld=770

\section{Enzymes}

$\mathrm{N}$-acylsphingosine amidohydrolase 2

http://www.guidetopharmacology.org/GRAC/ObjectDisplayForward?objectld=2492

$\mathrm{N}$-acylsphingosine amidohydrolase $2 \mathrm{~B}$

http://www.guidetopharmacology.org/GRAC/ObjectDisplayForward?objectld=2493

Alkaline ceramidases

http://www.guidetopharmacology.org/GRAC/FamilyDisplayForward?familyld=768

Enzymes

alkaline ceramidase 1

http://www.guidetopharmacology.org/GRAC/ObjectDisplayForward?objectld=2468

alkaline ceramidase 2

http://www.guidetopharmacology.org/GRAC/ObjectDisplayForward?objectld=2469

alkaline ceramidase 3

http://www.guidetopharmacology.org/GRAC/ObjectDisplayForward?objectld=2470

Ceramide kinase

http://www.guidetopharmacology.org/GRAC/FamilyDisplayForward?familyld=771

Enzymes

ceramide kinase 


\section{References}

1. Adam-Klages S, Adam D, Wiegmann K, Struve S, Kolanus W, Schneider-Mergener J and Krönke M. (1996) FAN, a novel WD-repeat protein, couples the p55 TNF-receptor to neutral sphingomyelinase. Cell 86: 937-47 [PMID:8808629]

2. Beauchamp E, Tekpli X, Marteil G, Lagadic-Gossmann D, Legrand P and Rioux V. (2009) N-Myristoylation targets dihydroceramide Delta4-desaturase 1 to mitochondria: partial involvement in the apoptotic effect of myristic acid. Biochimie 91: 1411-9 [PMID:19647031]

3. Butters TD, van den Broek LAGM, Fleet GWJ, Krulle TM, Wormald MR, Dwek RA and Platt FM. (2000) Molecular requirements of imino sugars for the selective control of $\mathrm{N}$-linked glycosylation and glycosphingolipid biosynthesis. Tetrahedron: Assymetry 11: 113-124

4. Camacho L, Simbari F, Garrido M, Abad JL, Casas J, Delgado A and Fabriàs G. (2012) 3-Deoxy-3,4dehydro analogs of XM462. Preparation and activity on sphingolipid metabolism and cell fate. Bioorg. Med. Chem. 20: 3173-9 [PMID:22537678]

5. Chen $Y$ and Cao Y. (2017) The sphingomyelin synthase family: proteins, diseases, and inhibitors.Biol. Chem. 398: 1319-1325 [PMID:28742512]

6. Coant N, Sakamoto W, Mao C and Hannun YA. (2017) Ceramidases, roles in sphingolipid metabolism and in health and disease. Adv Biol Regul 63: 122-131 [PMID:27771292]

7. Deng X, Lin F, Zhang Y, Li Y, Zhou L, Lou B, Li Y, Dong J, Ding T and Jiang al al.. (2014) Identification of small molecule sphingomyelin synthase inhibitors. Eur J Med Chem 73: 1-7 [PMID:24374347]

8. Fabrias G, Muñoz-Olaya J, Cingolani F, Signorelli P, Casas J, Gagliostro V and Ghidoni R. (2012) Dihydroceramide desaturase and dihydrosphingolipids: debutant players in the sphingolipid arena. Prog. Lipid Res. 51: 82-94 [PMID:22200621]

9. Graf C, Klumpp M, Habig M, Rovina P, Billich A, Baumruker T, Oberhauser B and Bornancin F. (2008) Targeting ceramide metabolism with a potent and specific ceramide kinase inhibitor. Mol. Pharmacol. 74: 925-32 [PMID:18612076]

10. Han G, Gupta SD, Gable K, Niranjanakumari S, Moitra P, Eichler F, Brown Jr RH, Harmon JM and Dunn TM. (2009) Identification of small subunits of mammalian serine palmitoyltransferase that confer distinct acyl-CoA substrate specificities. Proc. Natl. Acad. Sci. U.S.A. 106: 8186-91 [PMID:19416851]

11. He Y, Selvaraju S, Curtin ML, Jakob CG, Zhu H, Comess KM, Shaw B, The J, Lima-Fernandes E and Szewczyk MM et al.. (2017) The EED protein-protein interaction inhibitor A-395 inactivates the PRC2 complex. Nat. Chem. Biol. 13: 389-395 [PMID:28135237]

12. Houben E, Holleran WM, Yaginuma T, Mao C, Obeid LM, Rogiers V, Takagi Y, Elias PM and Uchida Y. (2006) Differentiation-associated expression of ceramidase isoforms in cultured keratinocytes and epidermis. J. Lipid Res. 47: 1063-70 [PMID:16477081]

13. Koch J, Gärtner S, Li CM, Quintern LE, Bernardo K, Levran O, Schnabel D, Desnick RJ, Schuchman EH and Sandhoff K. (1996) Molecular cloning and characterization of a full-length complementary DNA encoding human acid ceramidase. Identification Of the first molecular lesion causing Farber disease. $J$. Biol. Chem. 271: 33110-5 [PMID:8955159]

14. Lahiri S and Futerman AH. (2005) LASS5 is a bona fide dihydroceramide synthase that selectively utilizes palmitoyl-CoA as acyl donor. J. Biol. Chem. 280: 33735-8 [PMID:16100120]

15. Laviad EL, Albee L, Pankova-Kholmyansky I, Epstein S, Park H, Merrill Jr AH and Futerman AH. (2008) Characterization of ceramide synthase 2: tissue distribution, substrate specificity, and inhibition by sphingosine 1-phosphate. J. Biol. Chem. 283: 5677-84 [PMID:18165233]

16. Li YL, Qi XY, Jiang H, Deng XD, Dong YP, Ding TB, Zhou L, Men P, Chu Y and Wang Rðet al.. (2015) Discovery, synthesis and biological evaluation of 2-(4-(N-phenethylsulfamoyl)phenoxy)acetamides (SAPAs) as novel sphingomyelin synthase 1 inhibitors. Bioorg. Med. Chem. 23: 6173-84 [PMID:26314925]

17. Mao C, Xu R, Szulc ZM, Bielawska A, Galadari SH and Obeid LM. (2001) Cloning and characterization of a novel human alkaline ceramidase. A mammalian enzyme that hydrolyzes phytoceramide. J. Biol. Chem. 
276: 26577-88 [PMID:11356846]

18. Mao C, Xu R, Szulc ZM, Bielawski J, Becker KP, Bielawska A, Galadari SH, Hu W and Obeid LM. (2003) Cloning and characterization of a mouse endoplasmic reticulum alkaline ceramidase: an enzyme that preferentially regulates metabolism of very long chain ceramides. J. Biol. Chem. 278: 31184-91 [PMID:12783875]

19. Miyake $Y$, Kozutsumi $Y$, Nakamura S, Fujita T and Kawasaki T. (1995) Serine palmitoyltransferase is the primary target of a sphingosine-like immunosuppressant, ISP-1/myriocin. Biochem. Biophys. Res. Commun. 211: 396-403 [PMID:7794249]

20. Mizutani Y, Kihara A and Igarashi Y. (2005) Mammalian Lass6 and its related family members regulate synthesis of specific ceramides. Biochem. J. 390: 263-71 [PMID:15823095]

21. Mlinar B and Corradetti R. (2003) Endogenous 5-HT, released by MDMA through serotonin transporterand secretory vesicle-dependent mechanisms, reduces hippocampal excitatory synaptic transmission by preferential activation of 5-HT1B receptors located on CA1 pyramidal neurons. Eur. J. Neurosci. 18: 155971 [PMID:14511335]

22. Mo M, Yang J, Jiang XC, Cao Y, Fei J, Chen Y, Qi X, Chu Y, Zhou L and Ye D. (2018) Discovery of 4Benzyloxybenzo[ d]isoxazole-3-amine Derivatives as Highly Selective and Orally Efficacious Human Sphingomyelin Synthase 2 Inhibitors that Reduce Chronic Inflammation in db/ db Mice. J. Med. Chem. 61: 8241-8254 [PMID:30074791]

23. Philipp S, Puchert M, Adam-Klages S, Tchikov V, Winoto-Morbach S, Mathieu S, Deerberg A, Kolker L, Marchesini N and Kabelitz D et al.. (2010) The Polycomb group protein EED couples TNF receptor 1 to neutral sphingomyelinase. Proc. Natl. Acad. Sci. U.S.A. 107: 1112-7 [PMID:20080539]

24. Rabionet M, van der Spoel AC, Chuang CC, von Tümpling-Radosta B, Litjens M, Bouwmeester D, Hellbusch CC, Körner C, Wiegandt $H$ and Gorgas $K$ et al.. (2008) Male germ cells require polyenoic sphingolipids with complex glycosylation for completion of meiosis: a link to ceramide synthase-3. J. Biol. Chem. 283: 13357-69 [PMID:18308723]

25. Riebeling C, Allegood JC, Wang E, Merrill Jr AH and Futerman AH. (2003) Two mammalian longevity assurance gene (LAG1) family members, trh1 and trh4, regulate dihydroceramide synthesis using different fatty acyl-CoA donors. J. Biol. Chem. 278: 43452-9 [PMID:12912983]

26. Sun W, Xu R, Hu W, Jin J, Crellin HA, Bielawski J, Szulc ZM, Thiers BH, Obeid LM and Mao C. (2008) Upregulation of the human alkaline ceramidase 1 and acid ceramidase mediates calcium-induced differentiation of epidermal keratinocytes. J. Invest. Dermatol. 128: 389-97 [PMID:17713573]

27. Tani M, lida $\mathrm{H}$ and Ito M. (2003) O-glycosylation of mucin-like domain retains the neutral ceramidase on the plasma membranes as a type II integral membrane protein. J. Biol. Chem. 278: 10523-30 [PMID:12499379]

28. Tani M and Kuge O. (2009) Sphingomyelin synthase 2 is palmitoylated at the $\mathrm{COOH}$-terminal tail, which is involved in its localization in plasma membranes. Biochem. Biophys. Res. Commun. 381: 328-32 [PMID:19233134]

29. Venkataraman K, Riebeling C, Bodennec J, Riezman H, Allegood JC, Sullards MC, Merrill Jr AH and Futerman $\mathrm{AH}$. (2002) Upstream of growth and differentiation factor 1 (uog1), a mammalian homolog of the yeast longevity assurance gene 1 (LAG1), regulates $\mathrm{N}$-stearoyl-sphinganine (C18-(dihydro)ceramide) synthesis in a fumonisin B1-independent manner in mammalian cells. J. Biol. Chem. 277: 35642-9 [PMID:12105227]

30. Wang K, Xu R, Snider AJ, Schrandt J, Li Y, Bialkowska AB, Li M, Zhou J, Hannun YA and Obeid LNet al.. (2016) Alkaline ceramidase 3 deficiency aggravates colitis and colitis-associated tumorigenesis in mice by hyperactivating the innate immune system. Cell Death Dis 7: e2124 [PMID:26938296]

31. Xu R, Jin J, Hu W, Sun W, Bielawski J, Szulc Z, Taha T, Obeid LM and Mao C. (2006) Golgi alkaline ceramidase regulates cell proliferation and survival by controlling levels of sphingosine and S1P. FASEB J. 20: 1813-25 [PMID:16940153]

32. Yokomatsu T, Murano T, Akiyama T, Koizumi J, Shibuya S, Tsuji Y, Soeda S and Shimeno H. (2003) Synthesis of non-competitive inhibitors of sphingomyelinases with significant activity. Bioorg. Med. Chem. 
Lett. 13: 229-36 [PMID:12482429] 